\title{
Simulation of a large-eddy-break-up device (LEBU) in a moderate Reynolds number turbulent boundary layer
}

\author{
Cheng Chin · Ramis Örlü · Jason \\ Monty · Nicholas Hutchins · Andrew \\ Ooi · Philipp Schlatter
}

Received: date / Accepted: date

\begin{abstract}
A well-resolved large eddy simulation (LES) of a large-eddy breakup (LEBU) device in a spatially evolving turbulent boundary layer is performed with, Reynolds number, based on free-stream velocity and momentumloss thickness, of $R e_{\theta} \approx 4300$. The implementation of the LEBU is via an immersed boundary method. The LEBU is positioned at a wall-normal distance of $0.8 \delta$ ( $\delta$ denoting the local boundary layer thickness at the location of the LEBU) from the wall. The LEBU acts to delay the growth of the turbulent boundary layer and produces global skin friction reduction beyond $180 \delta$ downstream of the LEBU, with a peak local skin friction reduction of approximately $12 \%$. However, no net drag reduction is found when accounting for the device drag of the LEBU in accordance with the towing tank experiments by Sahlin et al. (Phys. Fluids 31, 2814, 1988). Further investigation is performed on the interactions of high and low momentum bulges with the LEBU and the corresponding output is analysed, showing a 'break-up' of these large momentum bulges downstream of the LEBU. In addition, results from the spanwise energy spectra show consistent reduction in energy at spanwise length scales for $\lambda_{z}^{+}>1000$ independent of streamwise and wall-normal location when compared to the corresponding turbulent boundary layer without LEBU.
\end{abstract}

C.Chin · J. Monty · N. Hutchins · A. Ooi

Department of Mechanical Engineering

University of Melbourne

Parkville, Victoria 3010, Australia

Tel.: +61-3-83443044

E-mail: chincc@unimelb.edu.au

R. Örlü · P. Schlatter

Linné FLOW Centre

KTH Mechanics

Royal Institute of Technology

SE-100 44 Stockholm, Sweden 
Keywords Boundary layer · Large eddy simulation · Wall turbulence • Large-eddy-break-up device · Drag reduction

\section{Introduction}

A paradigm shift in the understanding of wall turbulence took place once a certain degree of order was found to exist in the larger eddies of turbulent flows [1]. These organized, so-called coherent, structures were found to be ubiquitous in wall bounded turbulence with one class being associated with the wall layer [2] and another one with the outer layer of the boundary layer [3]. Furthermore, the coherent processes have been found to play a major role in the growth and evolution of turbulent boundary layers, thereby opening doors for the beneficial manipulation and control [4]. These discoveries lead to the birth of so called large-eddy break-up devices (LEBUs), which consist of one or more thin plates or airfoils placed parallel to the wall and immersed in the outer part of turbulent boundary layers. As their acronym suggests, these devices are believed to 'break up' the 'large-eddies' (Although other mechanisms have been suggested - refer to [5]). These devices were found to reduce the turbulence intensity for up to 100 local boundary layer thicknesses downstream of the device, and were capable of reducing the local skin friction and above all (seemingly) the net drag by substantial amount [6]. This result triggered an avalanche of follow-up studies [7,8, and reference therein], which, however, seemed to have faded away once direct drag measurements in towing tanks have shown that — while local and global skin friction reduction could be achieved - any substantial net drag reduction by means of LEBUs turned out to be implausible. As shown by Sahlin et al. [9], the device drag was found to exactly balance the local skin friction reduction. Despite the decline of interest in LEBUs or other outer layer devices (OLDs), due to their failure in delivering the promised net drag reduction, experimental investigations continued to explore their capability in locally reducing skin friction and turbulence $[10,11]$. So for instance have LEBUs been utilised for flow control strategies in the area of aero-optics, whereby it is reported that up to $33 \%$ aero-optical distortion reduction is achieved [12]. Another application of LEBUs in flow control is in the area of heat transfer, where augmentation of the wall heat transfer is obtained [13].

With the renewed interest in the very large-scale motions (VLSMs) [14] and their influence that extends to the wall [15], re-examination of LEBUs (and other OLDs) seems pertinent, especially given recent advances in our ability to simulate developing turbulent boundary layers. Indeed, despite the vast number of experimental investigations, there is, however, a clear lack of numerical simulations. Recalling the apparent experimental difficulties in accurately measuring the skin friction and drag in external flows, direct numerical simulations (DNS) would indeed be the perfect tool to provide the full details needed to understand the underlying mechanisms involved in turbulence and skin friction and possibly net drag reduction. Computer resources have, however, only over 
the last years become powerful enough to perform such simulations at relevant Reynolds numbers. One - and to the authors knowledge the only — work in this respect is the DNS by Spalart et al. [16], besides an early large eddy simulation (LES) [17]. Despite their pioneering character, they were, however, limited to low Reynolds numbers, where the inner and outer layer are barely discernible, and the observations made might not be transferable to higher Reynolds numbers. Recently, the Reynolds numbers achievable in turbulent boundary layers by means of DNS [18] and well-resolved large-eddy simulations (LES) [19] have increased considerably and call for a reconsideration of the flow around a LEBU at a numerically high Reynolds number.

\section{Numerical setup}

The numerical simulations involve two separate setups. The first setup is for the reference case, which is an evolving turbulent boundary layer (herein we will denote the reference case as TBL). The turbulent boundary layer LES is performed using a numerical scheme that is based on a fully spectral method [20] with an ADM-RT [21] subgrid-scale model. Here the streamwise, wallnormal and spanwise directions are denoted as $x, y$ and $z$ with corresponding velocities represented as $U+u, V+v$ and $W+w$. The inlet boundary condition is set to be a laminar Blasius boundary layer profile with $R e_{\delta_{o}^{\star}}=450$, where $\delta_{o}^{\star}$ is the displacement thickness at the inlet of the computational domain. A low amplitude forcing is imposed close to the inlet to trip the flow in order to achieve turbulent transition earlier (for details see [22]). The computational domain is $L_{x} \times L_{y} \times L_{z}=6000 \delta_{o}^{\star} \times 200 \delta_{o}^{\star} \times 240 \delta_{o}^{\star}$ in the streamwise, wall-normal and spanwise directions respectively as shown in figure 1 . The associated number of spectral collocated points is $6144 \times 513 \times 512$. The wall-parallel directions are discretized using Fourier series and the wall-normal direction is discretized with Chebyshev polynomials. The wall-parallel directions use dealiasing using the well-known $3 / 2$ rule, increasing the effective resolution in physical space. The temporal discretisation is a mixed Runge Kutta/Crank Nicolson scheme of third order. An adaptive time step is employed to ensure the Courant number is below 0.8. Table 1 shows a summary of the computational parameters.

The second setup is with a LEBU in the turbulent boundary layer. The second simulation essentially just imposes the LEBU device into the first setup. This is achieved using an immersed boundary method, which has previously

\begin{tabular}{ccccc}
\hline Case & $R e_{\theta}$ & $\Delta_{x}^{+}, \Delta_{y}^{+}, \Delta_{z}^{+}$ & $L_{x}, L_{y}, L_{z} / \delta_{99, L E B U}$ & $T U / \delta_{99, L E B U}$ \\
\hline TBL & $500-4300$ & $17.8,[0.034,11.23], 8.58$ & $272,23,11$ & 1000 \\
LEBU & $500-4300$ & $18.4,[0.035,11.37], 8.69$ & $272,23,11$ & 550 \\
\hline
\end{tabular}

Table 1 Summary of simulation parameters. $\Delta_{x}^{+}, \Delta_{y}^{+}$and $\Delta_{z}^{+}$are calculated using the global average skin friction velocity $u_{\tau, g}$. 
been successfully implemented using the same numerical scheme [23]. The LEBU is implemented in the boundary layer at the location $x=1000 \delta_{o}^{\star}, y=$ $17.6 \delta_{o}^{\star}$ (shown in figure 1), where the local boundary layer thickness is $\delta \approx$ $22 \delta_{o}^{\star}$. This corresponds to a wall-normal distance based on the local $99 \%$ boundary layer thickness of $0.8 \delta_{99}$, where the majority of experimental studies had placed their LEBU $[4-6,8,24-26]$. This wall-normal location is often reported to produce optimum skin friction drag reduction. The LEBU is modelled as a flat plate with length $L_{L E B U} \approx 0.9 \delta\left(20 \delta_{0}^{\star}\right)$, thickness of $T_{L E B U} \approx 0.0075 \delta\left(0.165 \delta_{o}^{\star}\right)$, and with infinite spanwise width. This gives an aspect ratio $(L / T)$ of approximately 120 .

At each timestep, a forcing term is computed and imposed to ensure that the velocities within the grid points in the LEBU reduce to zero. Figure 2(a) shows the forcing function around the LEBU. The amplitude $\left(A_{f}\right)$ of the forcing is applied via a smoothing function around the surface of the LEBU for stability of the solver. The forcing function is outlined in the Appendix. Figure 2(b) shows the $x z$ plane of instantaneous streamwise velocity at the wall-normal location where the LEBU is located. The region occupied by the LEBU can clearly be identified by zero velocity, which confirms that the LEBU is correctly implemented.



Fig. 1 Computational domain of the turbulent boundary layer LES. The LEBU is imposed after a complete washthrough and performed as a separate simulation.

\section{Effect of LEBU on skin friction and drag}

First we present the mean velocity flow visualization of the two cases in figure 3. The reference case (TBL) is shown in figure 3(a), while figure 3(b) depicts the TBL with the imposed LEBU. The effects of the LEBU on the boundary layer downstream of the device can directly be observed, primarily as an impeded growth rate of the boundary layer. In figure 4, the normalised mean 

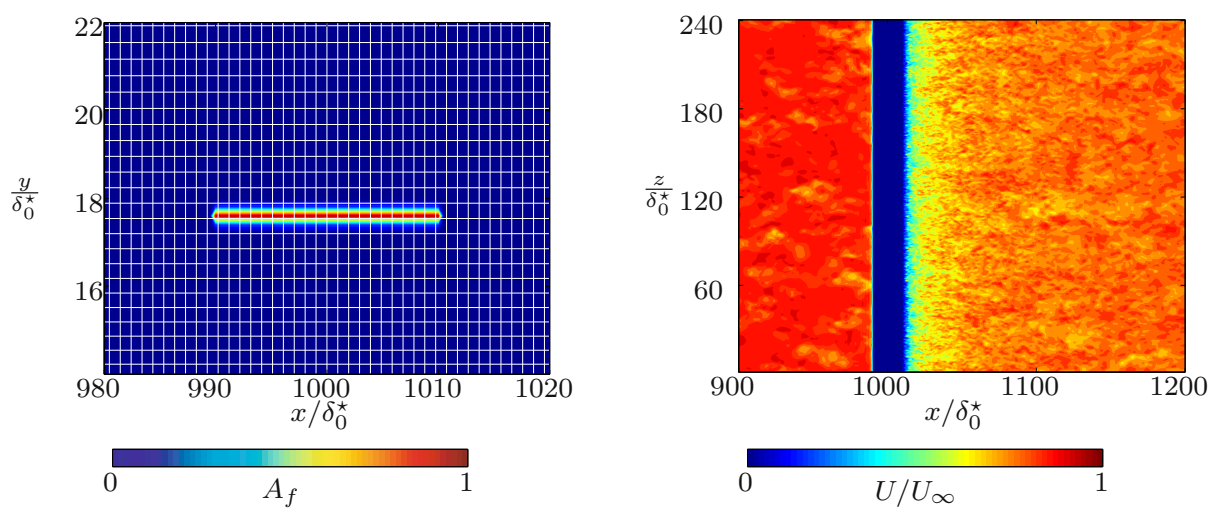

Fig. 2 (a) Amplitude of the forcing function around the LEBU. Red denotes a amplitude forcing of 1 and blue denotes no forcing applied. White lines indicate the wall-normal and streamwise grid spacings. (b) Instantaneous $(U+u) / U_{\infty}$ at the wall-normal location where the LEBU is located. i.e. $0.8 \delta_{99}$.

(a)



(b)

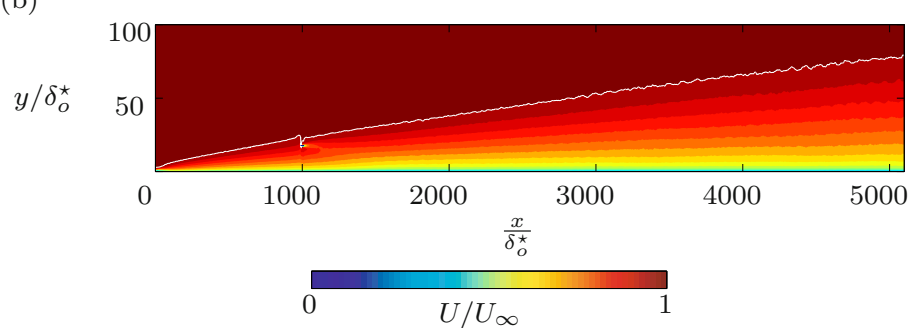

Fig. 3 Mean flow visualisation of $U$ for (a) TBL and (b) LEBU. White line indicates $\delta_{99}$.

velocity profiles $\left(U / U_{\infty}\right)$ at four different locations $(x / \delta \approx 1,5,50 \& 100)$ downstream of the LEBU are plotted, where $\delta$ denotes the boundary layer thickness at the location of the LEBU unless otherwise stated. The region immediately downstream the LEBU $(x / \delta \approx 1 \& 5)$ is clearly affected as apparent from the slight velocity deficit for the LEBU case when compared to the TBL case, which is due to the wake generated behind the LEBU. As expected, the velocity deficit in the wake region gradually diminishes with streamwise distance.

One of the well-known effects of a LEBU is the reduction in the skin friction as previously reported in literature $[2,16,24,25,5]$. Experimental reports have shown that a LEBU causes a decrease in the skin friction coefficient $c_{f}$ up to $120 \delta$ downstream of the LEBU [5]. Though it is not the primary aim of 



Fig. 4 Mean velocity profiles at four different locations downstream of the LEBU. Red lines denote TBL and blue lines are for the LEBU.
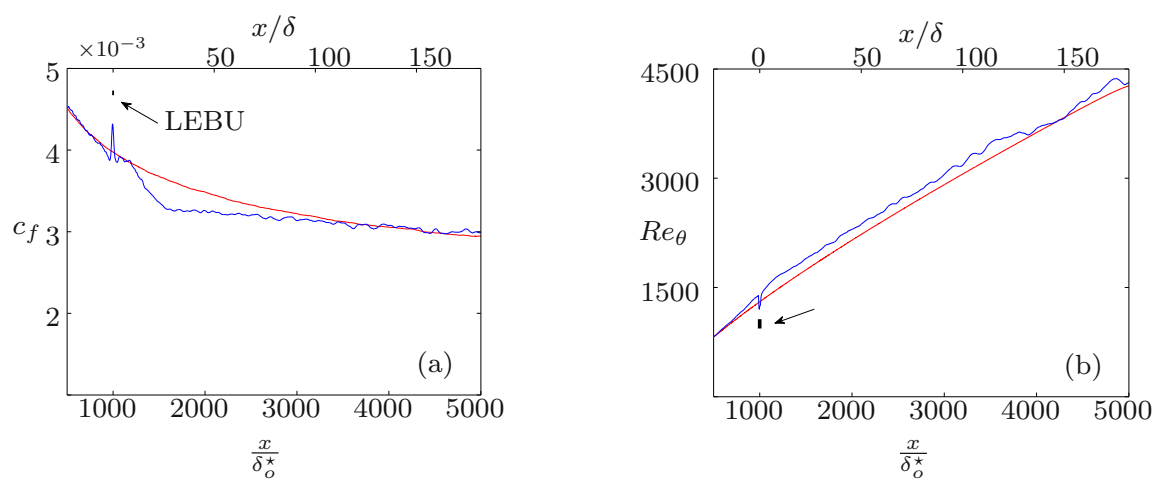

Fig. 5 Comparison of the (a) skin friction coefficient $c_{f}$ and (b) Reynolds number $R e_{\theta}$ based on free-stream velocity and momentum thickness between LEBU and TBL. Lines are as in figure 4.

this paper to investigate the best configuration of the LEBU (i.e. the dimension and location of the LEBU) for maximum skin friction drag reduction, an assessment of the performance of the current imposed LEBU on skin friction drag reduction is carried out. In figure 5(a), a comparison of the skin friction coefficient $c_{f}$ for the TBL (red line) and the LEBU case (blue line) shows a clear reduction in $c_{f}$ downstream of the LEBU. The error of $c_{f}$ for the LEBU case is within $\pm 1 \%$. The result shows a similar trend agreeing with the ex- 




Fig. 6 Percentage (\%) drag reduction verus streamwise distance $(x)$ for the LEBU against TBL. Positive +\% indicates drag reduction and negative $-\%$ denotes drag increase. Black line: Local skin friction drag reduction (SFR) at an instantaneous $x$ location; Blue line: Global skin friction drag reduction $(\overline{S F R})$; Red dash line: Average drag of the LEBU $\left(\overline{D_{L E B U}}\right)$; Red line: Net global drag reduction $(\overline{D R})$, calculated from $\sum$ (red dash line) + (blue line).

perimental studies mentioned earlier. The $c_{f}$ reduction seems to persist up to $3500 \delta_{o}^{\star}$, equivalent to $160 \delta$ downstream of the LEBU, which agrees with the skin friction reduction distance of $O(100) \delta$ reported in experiments [5]. The skin friction reduction is directly related to the streamwise gradient of the momentum thickness $\theta$ or $R e_{\theta}$ and in figure $5(\mathrm{~b})$, the $R e_{\theta}$ development is compared between the TBL and LEBU. Here the result shows that the LEBU case has a consistently higher $R e_{\theta}$ corresponding to a higher $\theta$ downstream of the LEBU. It is interesting to note that at a streamwise location of $x / \delta_{o}^{\star} \approx 4800$, the LEBU case appears to converge back to the TBL, which corresponds to the $c_{f}$ curve (figure $5 \mathrm{a}$ ), for which the LEBU case approaches that of the TBL. An advantage of this numerical study is the accurate analysis of the overall net global drag reduction $(\overline{D R})$ due to the LEBU, which would be difficult to obtain with similar accuracy in experiments. The $\overline{D R}$ is defined as the sum of the skin friction drag reduction due to the LEBU and the drag imposed on the LEBU shown in equation (1). The global skin friction reduction is denoted as $\overline{S F R}$ given as equation 2 and the average drag on the LEBU is labelled $\overline{D_{L E B U}}$, equation 3 . The local skin friction reduction $(S F R)$ is defined by equation 4 . The drag on the LEBU $\left(D_{L E B U}\right)$ is calculated using the streamwise momentum balance integral shown in equation (5) and illustrated in figure 7 . Here $T$ indicates the top of the control volume and $L$ is the corresponding length considered.

$$
\overline{D R}=\overline{S F R}-\overline{D_{L E B U}},
$$






Fig. 7 Control volume analysis for anaylsing drag due to the LEBU.

where,

$$
\begin{gathered}
\overline{S F R}=\underbrace{\frac{1}{x} \int_{0}^{x}\left(\tau_{w, T B L}-\tau_{w, L E B U}\right) \mathrm{dx}}_{\text {skin friction drag reduction }}, \\
\overline{D_{L E B U}}=\underbrace{\frac{1}{x} \int_{0}^{L}\left(D_{L E B U}\right) \mathrm{dx}}_{\text {Drag on LEBU }},
\end{gathered}
$$

and

$$
\begin{gathered}
S F R=\tau_{w, T B L}(x)-\tau_{w, L E B U}(x), \\
D_{L E B U}=\int_{0}^{T}\left(\Delta P+\Delta U^{2}\right) \mathrm{dy}+\int_{0}^{L}\left(U_{T} V_{T}+\overline{u v}_{T}-\tau_{w}\right) \mathrm{dx} .
\end{gathered}
$$

Figure 6 shows the percentage of drag reduction due to the LEBU with respect to the TBL. Positive and negative values denote drag reduction and increase, respectively. The black line represents the local LEBU $c_{f}$ reduction (SFR), which is compared to the TBL $c_{f}$ at the same $x$ location. The maximum $c_{f}$ reduction for the LEBU is approximately $12 \%$ at $25 \delta$ downstream of the LEBU, which is consistent with the results reported for a single LEBU $[17,5]$. Analysing the local $c_{f}$ reduction is potentially misleading, as the skin-friction reduction varies with streamwise distance. The blue line shows the global skin friction reduction $(\overline{S F R})$, which is the streamwise integrated $c_{f}$ reduction from the LEBU to a specific $x$ location downstream. The integrated $c_{f}$ reduction for the computational domain considered in this study is approximately $4 \%$. It is interesting to note that the global $c_{f}$ reduction persists across the entire streamwise domain even though the LEBU $c_{f}$ profile no longer indicates any local skin friction reduction. Next we analyze the net drag reduction shown by the red line $(\overline{D R})$. Initially there is a large net drag increase due to the device drag of the LEBU. The net drag gradually decreases with streamwise distance due to the rise in global $c_{f}$ reduction $(\overline{S F R})$. The drag on the LEBU without considering $c_{f}$ is shown as the red-dash line in the same figure. However at 
approximately $120 \delta$ downstream of the LEBU, the net drag reaches zero, suggesting that there is no net drag reduction due the LEBU, agreeing with the towing tank experiments by Sahlin et al. [9]. It is evident that the net drag is highly dependent on the domain length considered, which could potentially be a source for the discrepancies reported in previous studies. At this point, it is worth recalling that most of the classical experimental studies positioned their LEBU device at exactly the same wall-normal position, i.e. $0.8 \delta_{99}[4,6$, $8,24-26,5]$. Although some references indicate that the optimal location for drag reduction moves closer to the wall with increasing Reynolds number [27, 28], this observation could not be confirmed in the study by Sahlin et al. [9], which tested various heights in the range $0.4-0.9 \delta_{99}$.


Fig. 8 Instantaneous isocontour of $0.95 U / U_{\infty}$ shaded by $u / U_{\infty}$ for (a) TBL and (b) LEBU. 



Fig. 9 (a) Instantaneous $u / U_{\infty}$ of the LEBU simulation showing low momentum region in front of the LEBU. Black line indicates the LEBU. (b) Illustration of the von Kármán vortex street downstream of the LEBU.

\section{The LEBU as an input/output device}

One focus of the present study is to deepen our understanding of the feasibility of utilizing LEBUs as control devices. Figure 8 shows the instantaneous isocontour of $U / U_{\infty}=0.95$ and shaded by $u / U_{\infty}$. It is apparent from the figures that the flow features prior to the LEBU looks similar to the TBL. Upon interaction with the LEBU, a wake is generated from the LEBU, which introduces small-scale turbulence into the outer region of the boundary layer, and it is evident that large-scale motions appear to diminish after the LEBU. To investigate the effects of the LEBU better, we analyse the instantaneous fluctuating velocity $u$ at a zoomed-in region surrounding the LEBU device. Figure 9(a) shows a $x y$ plane of instantaneous $u$ around the LEBU. This location is chosen to illustrate regions of low momentum (figure 9a) in front of the LEBU. Shedding of vortices is visible in figure 9(a), which is further highlighted in figure 9 (b) by the presence of the von Kármán vortex street. This suggests that the LEBU adds small scale vorticity to the wake of the boundary layer. To study the interaction of these bulges with the LEBU, conditional averages of spatial and temporal data can be performed based on a positive large-scale fluctuation impacting on the LEBU, effectively analysing the LEBU as an input/output device.

Figure 10 shows the comparison of conditional averaged $u$ of the high momentum bulges with and without the LEBU (low momentum conditional averaged results are not shown as they are similar). The criteria for the conditional average is based on starting from a distance of $1 \delta$ upstream of the leading edge of the LEBU and then taking a straight line $L_{b}$ of $1 \delta$ further upstream. Only straight lines where the entire length of $L_{b}=1 \delta$ is of consistent $\pm u$ are taken, i.e. the value of $\pm u$ does not matter as long as $u$ is consistently + or - . Straight lines $L_{b}$ consisting of both $\pm u$ are discarded from the conditional averages. A total of 80 fields were used to compute the conditional averaged $\pm u$ with approximately $150 x y$ planes each for $+u$ and $-u$ per flow field. Figures 10(a,b) show the conditional averaged high momentum regions for the TBL and LEBU respectively. Once these streamwise-wall normal ( $x y$ ) planes are identified, these same $x y$ planes are averaged after interacting and convecting approximately $3 \delta$ and $7 \delta$ downstream of the LEBU. Figures $10(\mathrm{c}, \mathrm{d})$ 



(d)


Fig. 10 Conditional average of $u / U_{\infty}$ based on high momentum at upstream location of the LEBU. The first column represents TBL and the second is for the LEBU. $(a, b)$ High-momentum region upstream in front of the LEBU. (c,d) High-momentum region after convecting $x \approx 3 \delta\left(x / \delta_{o}^{\star} \approx 60\right)$ and $(\mathrm{e}, \mathrm{f})$ high-momentum region after convecting $x \approx 7 \delta$ $\left(x / \delta_{o}^{\star} \approx 150\right)$ downstream of the LEBU location. Black line represents the LEBU.

show the corresponding averages of figures $10(\mathrm{a}, \mathrm{b})$ after $3 \delta$ downstream of the LEBU and figures $10(\mathrm{e}, \mathrm{f})$ show the averages after $7 \delta$ downstream. It is evident from figure 10(d) that the LEBU serves to 'break-up' these large high momentum regions into two separate bulges whereas these high momentum regions remain as one (figure 10c) for the TBL as they convect downstream.

Next, we analyse the effects of the LEBU on other turbulence statistics downstream of the LEBU. Figure 11 (first column) compares the streamwise variance $u^{\prime+2}$ for the TBL and LEBU at various locations $x / \delta \approx 5,10,50$ and 180 downstream of the LEBU. One should note that the peak value of $u^{\prime+2}$ for the present LES is approximately $5 \%$ lower than the true peak $u^{\prime+2}$ value as e.g. obtained through DNS, cf. Ref. [19]. Since both the TBL and LEBU cases are exposed to the same attenuation effects, the associated discussions and findings are not biased by these subtle differences. The corresponding friction Reynolds number at these streamwise locations are $R e_{\tau}=U_{\tau} \delta_{99} / \nu \approx 490,530$, 740 and 1330 . We have chosen to scale the turbulence intensity with the friction velocity $U_{\tau}$ of the TBL (symbol '+' denote scaling with TBL friction velocity unless stated otherwise), as using a constant scaling allows comparison of the raw velocity fluctuations between TBL and the LEBU case. Note that scaling with $U_{\infty}$ would produce similar results. At $x / \delta \approx 5$, the wake generated by the LEBU causes a rise in the turbulence intensity at the wall-normal location where the LEBU is implemented. The near-wall peak seems unaffected at this streamwise location, possibly because the effect of the wake has not propagated to the near-wall region. This agrees with the $c_{f}$ results (figure 6) whereby 



Fig. 11 Comparison of (first column) the streamwise variance and (second column) intermittency at stream wise locations $x / \delta \approx 5,10,50$ and 180 (descending rows respectively) downstream of the LEBU. Red lines denote TBL and blue lines are for the LEBU. The black dash lines denote the wall-normal location of the LEBU.

the $c_{f}$ remains largely unaffected at this streamwise location. At streamwise locations $x / \delta \approx 10$ and 50 , the result from the LEBU case (blue line) clearly 
shows an attenuation of energy in the near-wall region as compared to the TBL (red line). A corresponding local $c_{f}$ reduction is also found at these locations. At a distance far downstream of the LEBU at $x / \delta \approx 180$, the LEBU turbulence intensity profile in the near-wall region seems to approximately collapse back to the TBL profile with slight difference observed in the outer region. Figure 11 (second column) shows the intermittency profiles at the same streamwise locations as the first column. Intermittency in this study is defined as intermittent existence of rotational and irrotational motion at the edge of the boundary layer. The intermittency for the boundary layer is calculated following the procedures outlined in Ref. [29]. The following turbulent kinetic energy equation (6) is used to identify the boundary layer interface.

$$
k=\frac{1}{U_{\infty}^{2}}\left[\left(U+u-U_{\infty}\right)^{2}+(V+v)^{2}+(W+w)^{2}\right],
$$

where $k$ is the turbulent kinetic energy. A threshold of $k=0.02$ is employed to identify the boundary layer interface. Results clearly show that in the near vicinity behind the LEBU, the wake generated causes the intermittency to rise at the edge of the boundary layer (indicating a higher probability of residence within a turbulent region). This is due to small-scale turbulence introduced by the wake, which has a persistent effect downstream of the LEBU. The intermittency plots at $x / \delta \approx 10$ and 50 support this, whereby the intermittency profile for the LEBU remains higher for all wall-normal locations. This can be interpreted as a reduction in the interface bulging, as shown in figure 8 . Further downstream $(x / \delta \approx 180)$, the intermittency profile of the LEBU collapses back to the TBL.

Though the variance profiles indicate a reduction in turbulence energy in the near-wall region, there is no information regarding how the energy carrying length-scales are affected. To examine the streamwise turbulence intensity further, we analyse the energy spectra, which provides information regarding the length-scale energy contribution to the overall turbulence energy.

Figure 12 (first and second column) show the spanwise premultipled energy spectra $\Phi_{u u}^{+}$of $u$ at the same locations as for those shown in figure 11 for the TBL and the LEBU case respectively. In both columns, one immediately notices a distinct peak in the energy spectra for TBL and LEBU. This is indicated by a white star ' $\star$ ' symbol located at $y^{+} \approx 15, \lambda_{z}^{+} \approx 120$. This is the well-known near-wall energetic peak due to the near-wall cycle. The vertical dashed line denotes the wall-normal location of the LEBU. In figures 12(b,e), the LEBU caused the energy present at its wall-normal location for the TBL case to shift to small length scales $\lambda_{z}^{+}$. This is consistent with the 'break-up' of large eddies to smaller scales as observed in figures 8 and 10, as well as the shedding of small-scales energy in the LEBU wake as observed in figure 9 . In figure $12(\mathrm{~h})$, there is an emergence of a secondary peak in the LEBU case at a location of $y^{+} \approx 100, \lambda_{z}^{+} \approx 400$, which is absent in the TBL (cf. figure $12 \mathrm{~g}$ ). This secondary peak due to the LEBU is still evident at $x / \delta \approx 180$ in figure $12(\mathrm{k})$, at $y^{+} \approx 200, \lambda_{z}^{+} \approx 700$. These results clearly provide evidence that not only are the large-scale motions manipulated by the LEBU, the small-scale 




Fig. 12 Comparison of the spanwise premultiplied energy spectra $\Phi_{u u}^{+}$for (first column) TBL and (second column) LEBU $x / \delta \approx 5,10,50$ and 180 (descending rows respectively) downstream of the LEBU. The last column is the difference in the energy between the LEBU and TBL. Symbol white ' $~ *$ ' is at $y^{+} \approx 15, \lambda_{z}^{+} \approx 120$. The vertical dash-line denoted the wall-normal location of the LEBU, the horizontal dash-line is at $\lambda_{z}^{+}=1000$.

motions are equally affected. To obtain a clearer image of what length scales are affected, we have subtracted the energy spectra of the LEBU case from the TBL. Figure 12 (third column) shows this difference, red and blue contours indicate gain and reduction in energy respectively, after implementing the LEBU into the TBL. The horizontal dashed line is at $\lambda_{z}^{+} \approx 1000$. The initial effect of the LEBU seems to be local to the device, causing a decrease in the large-scale energy and increase in the small-scale energy. At greater distances downstream, this effect seems to disperse closer to the wall until at $x / \delta \approx 180$ (figure 121), we see a very pronounced region of diminished large-scale energy for $\lambda_{z}^{+}>1000$. 


\section{Conclusions}

A large eddy simulation of a LEBU device imposed in a turbulent boundary layer is performed. The LEBU is implemented at a wall-normal location of $0.8 \delta$ via an immersed boundary method and clearly shows a reduction in the overall skin friction drag. However, when device drag is accounted for, no evidence of net drag reduction could be found. In fact, in this particular case, the drag induced by the LEBU exactly balances the skin friction drag reduction, thereby confirming the results by Sahlin et al. [9] and other follow-up studies, see e.g. Lynn et al. [30]. Zero net drag occurs at a streamwise location of $x / \delta \approx 120$ downstream of the LEBU, which suggests that this is the minimum domain length required for numerical simulations and experiments for this particular LEBU setup. A domain length that is inadequate would result in net drag increase. A conditional average is performed based on high and low momentum bulges immediately upstream of the LEBU, along with the corresponding output at 3 and $7 \delta$ downstream of the trailing edge of the LEBU. These results are compared to the baseline case TBL. The results clearly show that the LEBU 'breaks' these high and low momentum bulges into two halves, which is as expected, with a diminished overall fluctuation magnitude. A spectra energy analysis reveals a shift in large-scale energy to small-scales immediately behind the LEBU, due to 'breaking-up' of large-scale eddies and also the generation of small-scale vorticity in the wake region. The effect of the LEBU is most pronounced at $x / \delta \approx 180$ downstream of the device, showing large-scale energy reduction from the near-wall to the outer region. Overall, results suggest that the LEBU is effective to some extent in manipulating the large-scale motions. The reason for the downstream $c_{f}$ reductions has not yet been determined. Though the present simulated configuration does not function as a drag reducing device, it is hoped that further analysis of these results will shed further light on the mechanism by which LEBUs interact with the turbulent structure and manipulate $c_{f}$ locally. Although no net drag reduction was found, it might in certain circumstances be beneficial to reduce locally the skin friction and/or turbulence activity (e.g. for noise reduction) and in those cases, it could easily be implemented by mounting it on surfaces of ship hulls and aeroplane fuselages. Future simulations and studies on various configurations of the LEBU (e.g. active rather than tandem LEBUs) will be targeted at achieving drag reduction. Such insight will eventually pave the way for more efficient control strategies, in particular, with respect to the recent interest in control strategies targeting the large-scale structures remote from the wall.

Acknowledgements This research was undertaken with the assistance of resources provided at the NCI NF through the National Computational Merit Allocation Scheme supported by the Australian Government. Computer time was also provided by SNIC (Swedish National Infrastructure for Computing). The authors also acknowledge the financial support of the Australian Research Council as well as the Lundeqvist foundation. 


\section{Appendix}

The smoothing function $(\chi)$ used to implement the LEBU is as shown below in figure 13. Inside the blue region where the LEBU is located, $\chi=0$. Within the yellow region are functions consisting of a cosine smoothing equation as indicated by the equation numbers.

Everywhere else, $\chi=0$

\begin{tabular}{|c|c|c|}
\hline (15) & (10) & (16) \\
\hline (11) & LEBU & (12) \\
\hline (13) & (9) & (14) \\
\hline
\end{tabular}

Fig. 13 The forcing function $(\chi)$ around the LEBU. Blue region denotes the LEBU where $\chi=1$. The yellow region consists of smoothing function denoted by the equation numbers. Outside of the yellow region, $\chi=0$.

$$
\begin{gathered}
\chi=1\left\{\begin{array}{l}
x \leq X_{c}+0.5 L_{L E B U} \\
x \geq X_{c}-0.5 L_{L E B U} \\
y \leq Y_{c}+0.5 T_{L E B U} \\
y \geq Y_{c}-0.5 T_{L E B U}
\end{array}\right. \\
\chi=0\left\{\begin{array}{l}
x \leq X_{c}-\left(0.5 L_{L E B U}+0.2\right) \\
x \geq X_{c}+\left(0.5 L_{L E B U}+0.2\right) \\
y \leq Y_{c}-\left(0.5 T_{L E B U}+0.2\right) \\
y \geq Y_{c}+\left(0.5 T_{L E B U}+0.2\right)
\end{array}\right. \\
\chi=-\cos \left(\frac{y-Y_{c}+0.5 T_{L E B U}+0.2}{0.2} \pi\right)+0.5\left\{\begin{array}{c}
x \leq X_{c}+\left(0.5 L_{L E B U}\right) \\
x \geq X_{c}-\left(0.5 L_{L E B U}\right) \\
y \leq Y_{c}-\left(0.5 T_{L E B U}\right) \\
y \geq Y_{c}-\left(0.5 T_{L E B U}+0.2\right)
\end{array}\right. \\
\chi=\cos \left(\frac{y-Y_{c}-0.5 T_{L E B U}}{0.2} \pi\right)+0.5\left\{\begin{array}{c}
x \leq X_{c}+\left(0.5 L_{L E B U}\right) \\
x \geq X_{c}-\left(0.5 L_{L E B U}\right) \\
y \geq Y_{c}+\left(0.5 T_{L E B U}\right) \\
y \leq Y_{c}+\left(0.5 T_{L E B U}+0.2\right)
\end{array}\right. \\
\chi=-\cos \left(\frac{x-X_{c}+0.5 T_{L E B U}+0.2}{0.2} \pi\right)+0.5\left\{\begin{array}{c}
x \leq X_{c}-\left(0.5 L_{L E B U}\right) \\
x \geq X_{c}-\left(0.5 L_{L E B U}+0.2\right) \\
y \leq Y_{c}+\left(0.5 T_{L E B U}\right) \\
y \geq Y_{c}-\left(0.5 T_{L E B U}\right)
\end{array}\right)
\end{gathered}
$$




$$
\begin{gathered}
\chi=\cos \left(\frac{x-X_{c}-0.5 T_{L E B U}}{0.2} \pi\right)+0.5\left\{\begin{array}{c}
x \geq X_{c}+\left(0.5 L_{L E B U}\right) \\
x \leq X_{c}+\left(0.5 L_{L E B U}+0.2\right) \\
y \leq Y_{c}+\left(0.5 T_{L E B U}\right) \\
y \geq Y_{c}-\left(0.5 T_{L E B U}\right)
\end{array}\right. \\
\chi=\left[-\cos \left(\frac{y-Y_{c}+0.5 T_{L E B U}+0.2}{0.2} \pi\right)+0.5\right] \cdot\left[-\cos \left(\frac{x-X_{c}+0.5 T_{L E B U}+0.2}{0.2} \pi\right)+0.5\right] \\
\left\{\begin{array}{c}
x \leq X_{c}-\left(0.5 L_{L E B U}\right) \\
x \geq X_{c}-\left(0.5 L_{L E B U}+0.2\right) \\
y \leq Y_{c}-\left(0.5 T_{L E B U}\right) \\
y \geq Y_{c}-\left(0.5 T_{L E B U}+0.2\right)
\end{array}\right.
\end{gathered}
$$$$
\chi=\left[-\cos \left(\frac{y-Y_{c}+0.5 T_{L E B U}+0.2}{0.2} \pi\right)+0.5\right] \cdot\left[\cos \left(\frac{x-X_{c}-0.5 T_{L E B U}}{0.2} \pi\right)+0.5\right]
$$$$
\left\{\begin{array}{c}
x \geq X_{c}+\left(0.5 L_{L E B U}\right) \\
x \leq X_{c}+\left(0.5 L_{L E B U}+0.2\right) \\
y \leq Y_{c}-\left(0.5 T_{L E B U}\right) \\
y \geq Y_{c}-\left(0.5 T_{L E B U}+0.2\right)
\end{array}\right.
$$

$$
\begin{aligned}
\chi=\left[\cos \left(\frac{y-Y_{c}-0.5 T_{L E B U}}{0.2} \pi\right)+0.5\right] \cdot\left[-\cos \left(\frac{x-X_{c}+0.5 T_{L E B U}+0.2}{0.2} \pi\right)+0.5\right] \\
\left\{\begin{array}{c}
x \leq X_{c}-\left(0.5 L_{L E B U}\right) \\
x \geq X_{c}-\left(0.5 L_{L E B U}+0.2\right) \\
y \geq Y_{c}+\left(0.5 T_{L E B U}\right) \\
y \leq Y_{c}+\left(0.5 T_{L E B U}+0.2\right)
\end{array}\right.
\end{aligned}
$$

$$
\begin{gathered}
\chi=\left[\cos \left(\frac{y-Y_{c}-0.5 T_{L E B U}}{0.2} \pi\right)+0.5\right] \cdot\left[\cos \left(\frac{x-X_{c}-0.5 T_{L E B U}}{0.2} \pi\right)+0.5\right] \\
\left\{\begin{array}{c}
x \geq X_{c}+\left(0.5 L_{L E B U}\right) \\
x \leq X_{c}+\left(0.5 L_{L E B U}+0.2\right) \\
y \geq Y_{c}+\left(0.5 T_{L E B U}\right) \\
y \leq Y_{c}+\left(0.5 T_{L E B U}+0.2\right)
\end{array}\right.
\end{gathered}
$$

where $X_{c}$ and $Y_{c}$ are the $x, y$ location of the centroid of the LEBU, $T_{L E B U}$ and $L_{L E B U}$ are the thickness and length of the LEBU respectively. 


\section{References}

1. A.A. Townsend, The Structure of Turbulent Shear Flow (Cambridge University Press, Cambridge, 1956)

2. S.J. Kline, W.C. Reynolds, F.A. Shrub, P.W. Rundstadler, J. Fluid Mech. 30, 741 (1967)

3. G.L. Brown, A.S.W. Thomas, Phys. Fluids 20, 243 (1977)

4. T.C. Corke, Y. Guezennec, H.M. Nagib, Modification in drag of turbulent boundary layers resulting from manipulation of large-scale structures. Tech. Rep. 3444, NASA CR (1981)

5. A.M. Savill, J.C. Mumford, J. Fluid Mech. 191, 389 (1988)

6. T.C. Corke, H.M. Nagib, Y. Guezennec, A new view on origin, role and manipulation of large scales in turbulent boundary layers. Tech. Rep. 1165861, NASA CR (1982)

7. S. Tardu, G. Binder, Recent Developments in Turbulence Management pp. 147-160 (1991)

8. M. Walsh, J.J.B. Anders, Appl. Sci. Res. 46, 255 (1989)

9. A. Sahlin, A.V. Johansson, P.H. Alfredsson, Phys. Fluids 31, 2814 (1988)

10. V.I. Kornilov, Thermophys. Aeromech. 17, 249 (2010)

11. H. Park, N.H. An, N. Hutchins, K.S. Choi, H.H. Chun, I. Lee, J. Mar. Sci. Technol. 16 $390(2011)$

12. A.E. Smith, S. Gordeyev, AIAA Paper 2014-0321 (2014)

13. K. Inaoka, K. Suzuki, Turbulent Shear Flows 9, Springer-Verlag Berlin Heidelberg. pp. 365-382 (1995)

14. N. Hutchins, I. Marusic, J. Fluid Mech. 579, 1 (2007)

15. R. Mathis, N. Hutchins, I. Marusic, J. Fluid Mech. 628, 311 (2009)

16. P.R. Spalart, M. Streletsb, A. Travin, Int. J. Heat Fluid Flow 27, 902 (2006)

17. H. Klein, R. Friedrich, Turbulence Control by Passive Means 4, 41 (1990)

18. P. Schlatter, R. Örlü, J. Fluid Mech. 659, 116 (2010)

19. G. Eitel-Amor, R. Örlü, P. Schlatter, Int. J. Heat Fluid Flow 47, 57 (2014)

20. M. Chevalier, P. Schlatter, A. Lundbladh, D.S. Henningson, Simson - a pseudo-spectral solver for incompressible boundary layer flows. Tech. Rep. 7, Tech. Rep. TRITA-MEK (2007)

21. P. Schlatter, S. Stolz, L. Kleiser, Int. J. Heat Fluid Flow 25, 549 (2004)

22. P. Schlatter, R. Örlü, J. Fluid Mech. 710, 5 (2012)

23. M. Brynjell-Rahkola, P. Schlatter, A. Hanifi, D.S. Henningson, in 8th International Symposium on Turbulence and Shear Flow Phenomena (2013)

24. J.B. Anders, R. Watson, AIAA Shear Flow Control Conference 85, 0520 (1985)

25. G. Iuso, M. Onorato, Meccanica 30, 359 (1995)

26. M.W. Plesniak, H.M. Nagib, AIAA Shear Flow Control Conference 85, 0518 (1985)

27. J. Lemay, J. Delville, J.P. Bonnet, Exp. Fluids 9, 301 (1990)

28. J. Lemay, A.M. Savill, J.P. Bonnet, J. Delville, Turbulent Shear Flows 6, Springer-Verlag Berlin Heidelberg. pp. 179-193 (1989)

29. K. Chauhan, J. Philip, C. de Silva, N. Hutchins, I. Marusic, J. Fluid Mech. 742, 119 (2014)

30. T.B. Lynn, D.W. Bechert, D.A. Gerich, Exp Fluids 19, 405 (2004) 


\section{University Library}

\section{- M M N E R VA A gateway to Melbourne's research publications}

Minerva Access is the Institutional Repository of The University of Melbourne

Author/s:

Chin, C;Monty, J;HUTCHINS, N;Ooi, A;Orlu, R;Schlatter, P

Title:

Simulation of large-eddy-break-up device (LEBU) in a moderate Reynolds number turbulent boundary layer

Date:

2016-08-11

Citation:

Chin, C., Monty, J., HUTCHINS, N., Ooi, A., Orlu, R. \& Schlatter, P. (2016). Simulation of large-eddy-break-up device (LEBU) in a moderate Reynolds number turbulent boundary layer. Flow, Turbulence and Combustion, 98 (2), pp.445-460. https://doi.org/10.1007/ s10494-016-9757-y.

Persistent Link:

http://hdl.handle.net/11343/282710 